\title{
PROCESOS TRANSNACIONALES EN LAS REGIONES DE FRONTERA: LA TRIPLE FRONTERA EN EL SISTEMA MUNDO
}

\author{
Roberto Carlos Abinzano ${ }^{1}$
}

\begin{abstract}
Resumén: Las reflexiones que siguen son el resultado de una selección y abarcan temas diversos dentro de una única problemática general: los procesos transnacionales y la región de frontera. Desde hace dos décadas investigamos las regiones de frontera entre Argentina, Brasil y Paraguay. Definimos las regiones de frontera, diferenciándolas de las líneas de frontera, como un espacio transnacional que se estructura dinámicamente a partir de las relaciones de todo orden que tienen lugar, desde tiempos históricos de larga duración, entre agentes y colectivos de las tres naciones/estado. En este sentido nos apoyamos en la definición de Milton Santos sobre formación socio-espacial, pero, la aplicamos a una realidad transnacional espacialmente continua donde lo que interesaba era la determinación de un núcleo central constituido por una secuencia de formaciones sucesivas, integradas diacrónica y sincrónicamente y caracterizada por la existencia de redes complejas de relaciones muy antiguas y a la vez completamente nuevas.
\end{abstract}

Palabras-clave: transnacionalidad; fronteras, sistema mundo.

Abstract: The following reflections are the result of a selection and cover various topics within a single general problem: transnational processes and the frontier region. For two decades investigated border regions between Argentina, Brazil and Paraguay. We define the border regions, differentiating lines border as a transnational space that dynamically structure from relationships of all kinds that occur from long-term historical times between agents and groups from the three nations / State. In this regard we rely on the definition of Milton Santos on socio-spatial formation, but apply it to a transnational reality spatially continuous where we were interested in determining a core consisting of a sequence of successive formations, integrated diachronically and synchronically and characterized by the existence of complex networks of relationships completely new very old yet.

Keywords: transnationality; borders; world-system.

${ }^{1}$ Profesor Emérito Facultad de Humanidades y Ciencias Sociales Universidad Nacional de Misiones - Argentina. 
$|162|$

Processos transnacionales en las regiones de frontera:...

Desde hace dos décadas investigamos las regiones de frontera entre Argentina, Brasil y Paraguay. Definimos las regiones de frontera, diferenciándolas de las líneas de frontera, como un espacio transnacional que se estructura dinámicamente a partir de las relaciones de todo orden que tienen lugar, desde tiempos históricos de larga duración, entre agentes y colectivos de las tres naciones/estado. Hemos trabajado en dos épocas diferentes que se caracterizaron por la emergencia de procesos inéditos regionales. Son etapas que se corresponden con los tempos de la expansión y modalidades del capitalismo imperial y las redes financieras globalizadas.

El primero, a partir de mediados de los años ochenta que tuvo como marco la creación del MERCOSUR y su impacto local. Y el segundo, luego de los atentados a la AMIA y la embajada de Israel, potenciado luego por el "11/9", en el que la triple frontera pasa a integrar, para la ideología hegemónica imperial, "el eje del mal", soportando una ofensiva mediática internacional de gran envergadura y persistencia. Ambos procesos se articulan en una secuencia lógica y cronológica que reconoce etapas vinculadas a los megaprocesos de globalización, integración, formación de bloques, y durante la cual fue posible estudiar etnográficamente algunos procesos emergentes como : actividades económicas, mercado de trabajo, redes de movimientos sociales; redes de campesinos sin tierra; redes universitarias; movimientos ecologistas; organizaciones de pueblos originarios y foros en defensa de la región ante los embates imperiales.

Hasta ahora, la producción de nuestros proyectos de investigación se difundió através de, libros, de artículos en revistas científicas del país y el extranjero, en ponencias y comunicaciones, en conferencias, paneles, actividades académicas y en medios de comunicación masiva: artículos periodísticos, entrevistas, charlas, mesas redondas, etcétera.

Hoy queremos sintetizar en esta ponencia los aspectos centrales de un ciclo de investigaciones cuyos resultados todavía no fueron publicados en su totalidad y que constituyen el contenido de un libro que estamos concluyendo en estos momentos. Creemos 
que la importancia de los congresos consiste en hacer lo mas pública posible la producción universitaria, académica y profesional. Las reflexiones que siguen son el resultado de una selección y abarcan temas diversos dentro de una única problemática general: los procesos transnacionales y la región de frontera.

Cumplimos en cierto modo con lo aconsejado por Foucault en sus cursos:

“... puesto que nos pagan para investigar, ¿quién puede controlar las investigaciones que hacemos? ¿de qué manera podemos tener al corriente a quienes pueden interesarse en ellas y a los que tienen algún motivo de conectarse con ellas? ¿cómo se puede hacer, como no sea en definitiva, por la enseñanza, vale decir por la declaración pública, el informe público y mas o menos regular del trabajo que estamos realizando?" (Foucault, 2001: 15)

En este mismo sentido, cabe señalar que los avances de nuestras investigaciones se transmitieron en forma regular en el dictado de asignaturas de grado y postgrado en Antropología Social.

Comenzaremos con un breve racconto de los proyectos realizados hasta ahora y trataremos, en primer lugar de cuestiones teóricas y metodológicas. Nuestros proyectos estuvieron siempre vinculados a los movimientos sociales y organizaciones no gubernamentales de la región. Partimos desde Misiones hacia la región de frontera para alcanzar una visión superadora de las perspectivas particulares.

Hemos aspirado, y seguiremos haciéndolo, a contribuir al desarrollo de una conciencia sociopolítica y cultural que se oriente a la construcción de la integración de América Latina en una zona específica, en una época precisa y en un contexto internacional plagado de acechanzas, incertidumbres y guerras de opresión y saqueo. 
| $164 \mid$

Processos transnacionales en las regiones de frontera:...

Hay, en muchos de los procesos actuales de movilización popular, espontáneos o débilmente organizados, una búsqueda de participación no habitual dentro del sistema formal de nuestras débiles democracias formales carentes de un sólido contenido. Instituciones republicanas que habitan en un mar de contradicciones y arbitrariedades. Es cierto que muchos de estos fenómenos poseen un alto grado de espontaneísmo y utopismo. Pero las utopías pueden alimentarse con las ciencias para no ser inevitablemente la ratificación de su etimología: "en ninguna parte". Las ciencias sociales deben contribuir a la construcción de proyectos de inspiración utópica desde su praxis crítica y transformadora sin dejar, por eso, de incrementar el conocimiento. No alcanza con estudiar las fronteras con los modelos existentes, precisamente porque, como veremos mas adelante, las fronteras actuales implican la emergencia de lo nuevo, de los eventos inéditos, de nuevos sistemas y estructuras. Son esas emergencias las que suscitan nuestro máximo interés. Es decir, aquellos procesos que resultan de la interacción de las sociedades en contacto ante el impacto de la integración y el MERCOSUR. Se trata de cambios socioculturales, políticos y económicos de gran profundidad y, simultáneamente, de una intensa aceleración histórica. Para nosotros la frontera, como trazado liminar o línea de frontera, es solo un aspecto de las interacciones múltiples entre sociedades para cuyo estudio es imprescindible definir algunas categorías fundamentalmente relacionales y otras relativas a las dimensiones espaciales de las formaciones sociales, tan descuidadas por ciertas escuelas socio-antropológicas. Por eso, siguiendo a Milton Santos, hablamos de "formaciones socioeconómicas y espaciales". Su carácter histórico está comprendido en la categoría FSEE. Los sistemas socioculturales son impensables sin las dimensiones tiempo y espacio. Y el espacio es un factor de anclaje material de los procesos sociales por mas que las tendencias globalizantes hagan sentir su fuerza centrifuga incontenible. Lo local, el territorio, el lugar, el escenario físico, no pueden ser expulsados del análisis antropológico. 
Nuestra intención en este ciclo de investigaciones fue tratar las fronteras entre sociedades complejas y, por lo tanto, situarnos en los estudios de esta escala. Aunque debemos decir que el estudio de la sociedades complejas esta muy lejos de ser -en la actualidad- una especialidad de contornos definidos, ya que, toda la antropología moderna estudia sociedades complejas, se practica en sociedades complejas y a lo sumo focaliza su interés en fragmentos o segmentos de sociedades complejas a cuya totalidad debe necesariamente apelar. Y, además, debemos consignar, que no es lo mismo antropología de las sociedades complejas que "en las Sociedades complejas". Un estudio de las regiones de frontera debe combinar la etnografía de las situaciones de frontera concretas con una visión macro de la región tal como la definiremos en el presente texto y de las relaciones extra-regionales.

\section{La región de fronteras como objeto de estudio}

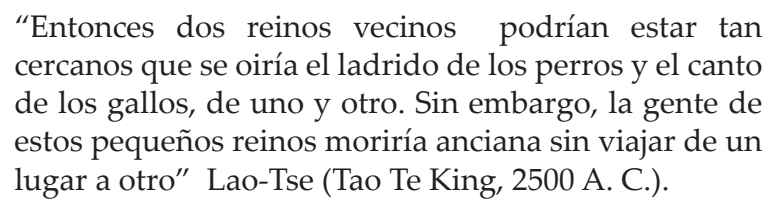

Nuestra experiencia en otras fronteras de America latina y del mundo fue el antecedente y el camino que nos llevó a las actuales investigaciones fronterizas y a una cierta especialización después de nuestra radicación en Misiones en 1978, durante la dictadura militar, hecho que consignamos porque las fronteras estaban vedadas para la curiosidad científica. Entonces existía en Misiones la filosofía de las fronteras defensivas bajo el marco de la doctrina de la seguridad nacional y la convicción que los amplios escenarios selváticos podían convertirse en ámbitos propicios para las guerrillas insurgentes, que en ese momento ya habían sido prácticamente diezmadas. 
|166 |

Processos transnacionales en las regiones de frontera:...

La preocupación mayor era el supuesto expansionismo de Brasil por medio del control de los ríos mediante la construcción de grandes represas hidroeléctricas y la presencia de población campesina intrusa. Se manejaban cifras como la relación de "20 a 1" de brasileños sobre argentinos en las zonas fronterizas. Se observaban con temor las migraciones riograndenses y la existencia de campesinos desplazados, sin tierras, que debían migrar por las buenas o por las malas ante el avance incontenible de la expansión sojera y la mecanización y modernización del agro.

La estrategia consistía entonces en fortalecer y poblar las áreas de frontera mediante algunos planes que nunca llegaron a concretarse. Veamos algunos ejemplos: a) Diagnóstico socioeconómico del "Área de Fronteras Bernardo de Irigoyen". b) "Plan de desarrollo para el área de frontera Bernardo de Irigoyen" que no tuvo ninguna concreción. c) "Proyecto para el desarrollo Integrado de la Provincia de Misiones" (1977/79). Todos proyectos y planes incumplidos en su mayor parte.

En 1979, y alejados de los equipos oficiales que realizaron los estudios mencionados, formulamos desde la universidad un proyecto destinado a conocer las fronteras desde una perspectiva antropológica comenzando con una tipología de diferentes situaciones: rural-rural, urbana-urbana y rural-urbana. Solo pretendíamos saber como funcionaban las fronteras realmente desde el punto de vista de sus pobladores.

La negativa a subsidiar estos estudios fue críptica, concluyente y obviamente negativa. La respuesta fue algo así como: el tema de las fronteras es para las instituciones de seguridad. Entre 1981 y 1985 redactamos una tesis doctoral consistente en un estudio sobre la formación de la sociedad provincial de Misiones que nos enfrentó, en primer lugar, a muchos vacíos historiográficos y a la convicción de que la compresión de esta sociedad era imposible analizar ciertos ciclos históricos en forma conjunta con lo ocurrido en el sur de Brasil y Paraguay. Fue entonces que debimos reflexionar sobre el concepto de Formación Social y su aplicación ampliada a una región internacional o espacio compartido por tres naciones desde tiempos remotos. Este enfoque estaba plagado de 
dificultades ya que cuando se hablaba de Formaciones Sociales los autores mas conspicuos se referían a etapas de naciones o imperios, pero no a regiones transnacionales de relaciones sistemáticas que generaban configuraciones sui generis. Tampoco nos servía la problemática de la etapa imperialista correspondiente a ese momento. Fue necesario, por lo tanto reflexionar sobre el concepto de región, tan trajinado en las ciencias sociales, para darle una nueva significación en el marco de la teoría de las formaciones socioeconómicas. En este sentido nos apoyamos en la definición de Milton Santos sobre formación socio-espacial, pero, la aplicamos a una realidad transnacional espacialmente continua donde lo que interesaba era la determinación de un núcleo central constituido por una secuencia de formaciones sucesivas, integradas diacrónica y sincrónicamente y caracterizada por la existencia de redes complejas de relaciones muy antiguas y a la vez completamente nuevas.

Se trataba de algo exploratorio que ofreció mejores resultados en algunas relaciones transfronterizas históricas o actuales que en otras. Por ejemplo, las tierras pobladas por aborígenes de la población Mbya de la familia Tupí-Guarini, que habitaban estas zonas en tiempos prehispánicos, constituyen una capa uniforme que es transnacional por naturaleza. Las aldeas Mbya existen en los tres países en numerosas parcialidades dispersas que están en contacto entre si y que suman aproximadamente unas 15.000 personas. Subjetivamente son los grupos mas trasnacionales de la frontera y su adscripción a uno u otro estado nación siempre es muy problemática y depende de sus estrategias adaptativas.

$\mathrm{Al}$ entrar en la órbita inglesa del capitalismo industrial y el liberalismo, en esta región se instaló en las tres naciones el sistema extractivista o Frente Extractivo en el área de fronteras, utilizando mecanismos y relaciones de producción precapitalistas por la ausencia de hecho de un salario real y la supervivencia de prácticas esclavistas. En pleno auge del sistema extractivista se constituyó, en toda la región compartida con Brasil y Paraguay, la colonización agrícola extranjera de origen europeo, que dio a toda la región transnacional "un aire de familia" que perdura. Este fue 
| 168 |

Processos transnacionales en las regiones de frontera:...

un proceso que se produjo en varias etapas, no solo cronológicas sino cualitativas y que hemos descrito detalladamente en otra parte (ABÍNZANO, 1985). Uno tras otro, estos sistemas socioculturales, sin desaparecer totalmente, salvo las reducciones jesuiticas (pero no sus improntas y secuelas) se articularon en el espacio y en el tiempo conformado un ciclo de formaciones sociales espaciales de carácter transnacional. Desde el Centro de Estudios para la Integración Latinoamericana (CEPIL), del cual fui director, se comenzó por fin abordar en forma ordenada y sin trabas políticas la cuestión de las fronteras en el marco del tratado de integración de Argentina con Brasil y el MERCOSUR, después. Tampoco puedo en este espacio resumir la obra de este centro porque prefiero centrarme en los estudios con mayor énfasis en lo etnográfico. Pero luego de realizar numerosas tareas vinculadas a la integración elegimos investigar las redes transfronterizas de movimientos sociales de los tres países y prestar nuestra cooperación y asistencia a dichas iniciativas espontáneas o semi-espontáneas surgidas ante la incertidumbre del impacto de la integración. Todo comenzó cuando el Movimiento Agrario Misionero, nos invitó a contribuir con información y capacitación en una serie de actividades que venían realizando con movimientos similares de Brasil y Paraguay. La concurrencia al primer encuentro que tuvo lugar en Santo Cristo en Río Grande Do Sul fue la piedra de toque de los proyectos que resumiremos mas adelante. Se llamó: Primer encuentro de trabajadores fronteirizos rurales. A partir de ahí encontramos el centro de gravedad de toda indagación posterior. Estos grupos de las tres naciones mas Uruguay, eran de origen popular, compuesto por pequeños productores rurales y peones, por grupos ecologistas y militantes de organizaciones de base, cooperativas, etc. y, con el apoyo de profesionales y asesores, demostró a lo largo de tres días de deliberaciones que la integración reconocía muchos caminos posibles, muchos modelos, y que frente a los proyectos cupulares se podía contraponer una alternativa diferente en lo ideológico, lo político y lo cultural. Era posible vislumbrar una integración basada en múltiples aspectos, protagonizada por todos los sectores de la comunidad y convertida en un campo de lucha de modelos 
y formas de vida enfrentados. Cabe señalar que nuestro interés no consistía en hacer una investigación de campesinado o productores rurales, sino de observar un fenómeno emergente. Una respuesta a las determinaciones extrarregionales en forma de contrapropuestas sui generis. Y, en lo posible, seguir paso a paso, etnográficamente, las acciones de esta red que se estaba conformando. ¿Qué es esto de la integración y el MERCOSUR? ¿En que puede afectarnos y en que beneficiarnos? Estas preguntas se hacían desde una posición de clase y en estas deliberaciones y durante los días de convivencia, las diferencias de idioma y nacionalidad quedaron en una plano secundario que, solo afloró como era obvio en la realización de un partido de futbol entre los brasileños, que eran locales en Santo Cristo, y el resto. Por suerte el partido terminó en un empate.

Entonces, una cosa era la integración en general o el Mercosur, y otra diferente las fronteras como realidades específicas en las que tenían lugar los sucesos estudiados. Debíamos avanzar todavía para elaborar un modelo de análisis y acotar mejor nuestro interés. Paralelamente se produjo un acercamiento entre los profesionales brasileños y paraguayos y esa circunstancia nos permitió crear una segunda red de universidades que hasta hoy esta dando sus frutos.

Pensando la frontera. No podemos resumir aquí las largas discusiones que sostuvimos para arribar a la selección de un número de categorías que nos sirvieran para delimitar los grandes campos de interés. Pero deseamos incluir a continuación una definición de los principales conceptos teóricos utilizados luego de una ardua búsqueda para compartir con el lector las herramientas utilizadas en nuestra concepción de las fronteras y la aplicabilidad de estos esquemas conceptuales a los estudios empíricos.

El término frontera a recibido una infinidad de definiciones de diversa índole. Es por eso que su redefinición y adecuación para nuestros estudios ha sido fundamental. Las sociedades humanas no poseen límites precisos como la piel del cuerpo humano. Los sistemas socioculturales son sistemas abiertos de inmensa complejidad.

Las formaciones sociales son en realidad -como dijimossocio-espaciales. Las fronteras entre pueblos son esencialmente 
| 170 |

Processos transnacionales en las regiones de frontera:...

simbólicas salvo en el caso de las líneas de frontera políticas y jurídicas donde es necesario al menos establecer un conjunto de referencias materiales concretas. Las fronteras antropológicas suponen la posibilidad de áreas de "mezcla sociocultural" entre diferentes sistemas en contacto. $\mathrm{Y}$ estas relaciones se producen traspasando las líneas de frontera. Podemos imaginar las aguas de un gran río que desemboca en el mar. Allí se mezclan las aguas dulces y saladas sin límites muy precisos porque se trata de fenómenos dinámicos. Vemos entonces que el concepto de frontera se proyecta sobre espacios-escenarios, modificados y modificantes, dinámicos y, por lo tanto, históricos, que conviene llamar, desde nuestra perspectiva, regiones de frontera. La línea de frontera es el límite político y jurídico concreto donde están situadas las instituciones dedicadas a controlar y regular los flujos transfronterizos. Estas instituciones trabajan sobre diferentes aspectos y constituyen una compleja red de prácticas muchas veces superpuestas y contradictorias que responden a diferentes intereses de la estructura burocrática. Del lado argentino encontramos, por ejemplo, gendarmería, prefectura, área de frontera, migraciones, aduana, sanidad, bromatología, etcétera. Cuanto mas rudimentaria es la integración entre dos países, mas instituciones existen en la línea de frontera, mas difíciles son sus relaciones y mas obsoletos parecen sus objetivos. Siempre sostuvimos que existe una relación dialéctica entre las fronteras de los mapas que marcan la soberanía territorial de la nación oficialmente objetivada en las instituciones mencionadas y lo que los pueblos hacen realmente en estas regiones. Obviamente, el interés socio-antropológico debe centrase en este ultimo termino de la relación, pero sin desconocer su relación con respecto al primer término. Tampoco hemos substancializado el concepto de frontera ni ninguna de las nociones conexas que complementan nuestras definiciones centrales. Nos quedan dudas respecto al valor relativo de trabajos que se realizan sin partir de una mínima definición de sus categorías para analizar un tema tan complejo partiendo de nociones de uso vulgar, metafórico, confusas y endebles. Y también nos produce cierto rechazo la pretensión de proyectar hacia las fronteras en general algunas 
conclusiones pertenecientes a trabajos empíricos muy localizados $\mathrm{y}$ restringidos a un tema en particular.

A veces, el razonable y justificado miedo a la reificación (cosificación, substancialización, etc.) desconoce la materialidad de los fenómenos a los que aluden las ideas con lo cual se cae en la adoración del ídolo del discurso per. se y en su inevitable consecuencia: el idealismo.

Denominamos región de frontera, a una noción socioantropológica que implica la presencia del factor espacial. Una región de frontera es un conjunto de sistemas socioculturales que se hallan localizados a ambos lados de una línea de frontera y que interactúan de múltiples formas. Al utilizar el término región sabemos que nos internamos en el debate sobre esta noción tan controvertida. Solamente queremos precisar que nuestra definición de región se basa en factores socio- antropológicos espacializados y en el concepto de formación socio-espacial. Lo que interesa de las regiones es el núcleo central del complejo sistema de interacciones de las praxis y no tanto sus límites precisos, como sostuvo alguna vez Pierre Georges.

Hay fenómenos que son nacionales y estructurales, como la inflación, pero que en una región particular tienen un comportamiento singular. La combinación específica de factores generales y locales otorga a la región su perfil, su singularidad. Todos los fenómenos macro-estructurales, nacionales y aun internacionales poseen una forma de ser específica, localizada. Nuestra región como ámbito "construido" incluye: a) la provincia de Misiones, el noreste de Corrientes, $b$ ) el borde occidental de Rio Grande Do Sul, Santa Catarina y Paraná, en Brasil y c) el este de Paraguay (desde el río homónimo).

Es decir que, aquello que intenta limitar la línea de frontera escapa en buena medida a su control. La línea de frontera es, por lo tanto un atributo interior a la region de fronteras, es un predicado incluido en el sujeto, es parte de la propia definición, muy importante, pero no el decisivo. La región de frontera es un espacio arbitrariamente establecido - un modelo heurístico hecho 
de hipótesis a ser contrastadas - y los fenómenos que ocurren en su interior están protagonizados por agentes sociales organizados en sistemas socioculturales abiertos, entrópicos, dinámicos. Estamos desechando todo uso metafórico del concepto frontera. Hablamos de fronteras entre naciones estado. Y, por lo tanto, de fronteras que señalan como cicatrices cartográficas la obra del colonialismo y la geopolítica de la hegemonía mundial que tuteló el surgimiento de nuestros estados. La región como proyecto es el gran laboratorio de la integración con los pueblos en contacto muy diferente a la integración abstracta de los tratados y los flujos comerciales. Tiene la potencialidad de generar proyectos propios y de rechazar imposiciones indeseables. Nunca en la capacidad de generar un polo contra-hegemónico desde la región pero si, creímos y creemos en las prácticas contra-hegemónicas múltiples algunas de las cuales provienen de enclaves regionales.

Dentro de la región denominamos área de frontera, al espacio de la región de frontera, perteneciente a uno de los dos países involucrados y en cuyo perímetro un segmento se corresponde con la línea de frontera . La dimensión total del área de frontera está a veces establecida oficial y arbitrariamente en kilómetros. Con el nombre de Área de Frontera fue creada una repartición burocrática oficial en Argentina destinada a coordinar a las instituciones específicas y realizar estudios y proyectos had hoc. Esta concepto corresponde al utilizado en Brasil como Faixa de Fronteira.

En cuanto al empleo del término patrones de asentamiento debemos consignar que el término fue usado primero en arqueología para referirse a la distribución regular y característica de los pueblos y sus actividades en el espacio. Tuvo valor en nuestros estudios históricos de la región, ya que las fronteras jurídicas se establecieron sobre una realidad caracterizada por la convergencia de diversos frentes que poseían distintos patrones de asentamiento. Este concepto nos ha servido para analizar muchos aspectos relativos a las formas de ocupación del espacio en áreas y regiones de frontera y está directamente vinculado a la noción de frentes de expansión y ocupación de espacio. El interés de esta noción y sus derivaciones metodológicas radica no solo en la inteligibilidad de procesos 
históricos sino en la expansión actual de muchos frentes hacia las fronteras.

El concepto de frente (frontis) alude a la máxima expansión territorial de una actividad humana como: frente agrícola, comercial, minero, extractivista y otros. En las situaciones de complejidad los frentes se dan generalmente combinados y articulados. Algunos autores denominan fronteras a lo que nosotros llamamos frentes.

Existen numerosos ejemplos históricos en los cuales varios frentes diferentes actuaron en forma combinada y complementaria ocupando los mismos espacios y estableciendo relaciones de competencia, articulación, colaboración o indiferencia. Por ejemplo, avanzadas militares combinadas con colonización, ocupación espontánea de tierras liberadas o baldías, colonización, extracción, etcétera. La relación entre frentes y fronteras es de la mayor importancia debido a que muchas fronteras, sobre todo en países nuevos, fueron establecidas en mesas de negociación diplomática y los territorios involucrados en la distribución fueron posteriormente ocupados en forma sistemática y dirigida o bien espontánea. En algunos casos, los frentes fueron posteriores a la demarcación jurídica de las líneas de frontera y, en otros, ocurrió lo contrario: primero avanzaron las sociedades sobre los espacios libres u ocupados por otras sociedades y después se procedió a consagrar los límites o líneas de fronteras. En América Latina se dieron ambos casos. Algunos pueblos del mismo origen, muy antiguo fueron separados por las convenciones entre estados naciones y otras regiones en cambio fueron repartidas sin que hubiera ninguna presencia de población de esos estados. Una vez trazados los mapas de soberanía se procedió a ocuparlos mediante campañas militares, generalmente genocidas o formas de colonización diversas. Todas las actividades que atraviesan las líneas de fronteras pueden ser considerados flujos. Denominamos flujos a las corrientes de desplazamiento de ciertos entes por el espacio que cruzan las líneas de frontera en ambas direcciones. En el caso de las fronteras internacionales interesan los desplazamientos de personas, de objetos (vehículos, mercancías, etcétera) y de mensajes simbólicos (radiales, televisivos, gráficos, de transmisión 
oral, etcétera, y de cualquier otra forma de información) Estos flujos pueden ser regulares, esporádicos o excepcionales. Técnicamente pueden ser descriptos, analizados, interpretados y ponderados mediante procedimientos cualitativos y cuantitativos. Por debajo de la definición abstracta de frontera se encuentra una cantidad infinita de realidades fronterizas diferentes que pueden agruparse en tipos de frontera o de situaciones de frontera. La diferencia entre estas nociones radica en que el tipo es mas estático y la situación mas dinámica y cambiante. Una frontera puede ser tipológicamente definible como urbana-urbana, por ejemplo, y al mismo tiempo atravesar diferentes etapas de cambio. Una tipología de las fronteras nos llevaría necesariamente a realizar en primer término, una distinción entre fronteras rurales, urbanas, mixtas, desérticas, superpobladas, infranqueables, permeables o impermeables, etc. Son categorías clasificatorias de rangos distintos. Lo ideal sería establecer tipos con fines heurísticos que combinaran todas estas categorías. La historia muestra numerosos intentos de construir barreras infranqueables que una a una fueron cayendo. En América Latina observamos que existen extensas fronteras donde no hay población ni controles y donde todavía hoy se circula con total libertad. Una mínima inspección a un mapa detallado muestra los inmensos vacíos que acompañan -por ejemplo- a la dilatada línea de frontera de Brasil con sus vecinos Paraguay, Bolivia, Perú, Colombia, Venezuela, Surinam, Guyana, etc. Hay fronteras, como dijimos en otro lugar, que "existen en los mapas" y donde es necesario investigar y descubrir la verdadera frontera entre las sociedades. Pero en algunos casos están en los mapas y no hay poblaciones en contacto porque no hay poblaciones de ningún tipo.

Fue así como diferenciamos la línea de frontera convencional entre estados naciones de las fronteras humanas concretas constituidas por las relaciones e interacciones que tenían lugar en una vasta región que denominamos "de frontera" y en cuyo interior se sitúa la línea de frontera, al solo efecto de reintegrar luego la totalidad de los fenómenos estudiados mediante modelos 
explicativos, descriptivos e interpretativos complejos. Las fronteras son las personas.

La frontera sociocultural es un entramado abierto de límites siempre imprecisos determinada por las cambiantes actividades humanas. Es el sistema de relaciones transfronterizas que tienen características sui generis porque están determinadas por su posición territorial. Se trata de procesos espacializados y arraigados en la materialidad de esas localizaciones. De manera que esta segunda concepción de frontera esta muy lejos de ser substancialista, ya que solo un idealista a ultranza podría atribuir a una definición que posee anclaje en la materialidad ese carácter que se le asigna.

Los flujos transfronterizos pueden ser de diversos tipos generales, a su vez divisibles en categorías desagregadas. Hay relaciones sistemáticas y permanentes; relaciones esporádicas y ocasionales; flujos de comunicación y mensajes; flujos erráticos y no recurrentes; flujos extrarregionales que utilizan región en transito;

Todos estos flujos pueden ser observados, descriptos, analizados, ponderados cualitativamente y cuantitativamente. Cuando los flujos son recíprocos y permanentes y constituyen interacciones basadas en ciertos objetivos o propósitos forman parte de nuestro interés, sobre todo cuando estas interacciones conforman fenómenos emergentes. Y muchos mas, obviamente, cuando alcanzan la complejidad de conformar redes de agentes

El concepto de red utilizado no reemplaza otras aproximaciones teóricas y metodológicas sino que las complementa. Por ejemplo, un estudio de conflicto de clases puede verse perturbado por la aplicación de análisis de redes pero también enriquecido.

Nuestro interés ha sido investigar ciertas redes de movimientos sociales y de organizaciones en la medida en estas expresaban instancias sociales y políticas emergentes que en si mismas eran movimientos sociales y políticos, heterogéneos y complejos. Pero no el único interés, ya que, debemos insistir en eso: 
$\mid 176$ |

Processos transnacionales en las regiones de frontera:...

la puerta de entrada a un objeto complejo debe serlo a la complejidad y no solamente a los recortes arbitrarios por bien fundados que estén. De manera que, cuando iniciamos un segundo ciclo de estudios destinados a conocer los nuevos fenómenos emergentes del impacto de la integración y el MERCOSUR partimos de la situación privilegiada de conocer la región transfronteriza, o transnacional, y de haber logrado aventurarnos en las realidades del sur de Brasil y el este de Paraguay potenciando ese conocimiento con ayuda, en muchos casos, de los investigadores de esos países a partir de una cooperación científica que fue también una consecuencia inmediata de la misma corriente integradora pero en el nivel del conocimiento, especialmente desde las universidades de la misma región de fronteras. Las demandas comunitarias al centro provenían de sectores muy disímiles: cámaras de comercio, instituciones oficiales, medios de comunicación, investigadores, docentes, estudiantes, industriales, funcionarios municipales, gremios, asociaciones culturales y deportivas, etcétera.

Los estudios realizados sobre los movimientos sociales y sus redes transfronterizas nos llevaron a un nuevo estudio que titulamos "Integración regional fronteriza. Sectores vulnerables frente al impacto global. Políticas y acciones alternativas", que intentó establecer la heterogeneidad del impacto y la detección de diferentes sectores por su grado de vulnerabilidad; las acciones llevadas adelante por estos segmentos a través de sus organizaciones, movimientos o representantes; la evaluación de sus estrategias; y fundamentalmente, la sociogénesis de nuevas redes, , sus acciones y estrategias, sus éxitos y fracasos. El escenario de estos procesos fue siempre la región de fronteras, ámbito cuya definición nos obligó a numerosos esfuerzos metodológicos y teóricos, y la red mencionada fue el núcleo de nuestro enfoque. Pudimos establecer en grandes líneas los factores que actuaban a favor o en contra de la consolidación y desarrollo de las redes.

El diagnóstico al que arribamos en relación a los factores señalados fue en síntesis:

A) Factores de cohesión: que contribuyen al desarrollo de la RED: 1) Preocupación por los problemas medioambientales. 
2) Interés por la difusión de información y cooperación en relación a las redes de agricultura orgánica ya existentes, así como el deseo de su ampliación. 3) Desarrollo de programas conjuntos de agricultura integral con la inclusión de temas como: agroindustrias, comercialización directa, utilización de técnicas no agresivas para los ecosistemas, ferias francas, etc. 4) Planes de créditos rotativos bajo la cobertura de sistemas mutuales o cooperativos. 5) Creación de empresas, instituciones u Ongs binacionales o trinacionales. 6) Lucha contra la construcción de nuevas empresas hidroeléctricas. 7) Defensa de los derechos humanos, de la mujer. De la infancia, etcétera 8) Defensa del modo de vida campesino, del acceso a la tierra y la titularidad de la tierra. 9) Búsqueda de fuentes de información, capacitación y organización mas eficaces. 10) Confianza creciente en las ONG e instituciones de apoyo, como las universidades en procura de fortalecer sus prácticas. 11) Demanda de ayuda al estado (nacional, provincial o municipal o sus equivalentes de Brasil y Paraguay) 12) Rechazo total o parcial del modelo neoconservador y los ajustes económicos.

B) Factores de conflicto: o que no contribuyen al desarrollo de la red. 1) Procesos políticos internos (nacionales) que absorbieron las prácticas de los principales militantes de las ONG, sindicatos, etc. De mayor relevancia, sobre todo en los procesos electorales. 2) Diferentes visiones de los problemas en cuanto a los contextos mayores que los determinan. 3) Diferentes grados de formación y compromiso de los militantes, líderes o delegados. 4) Problemas surgidos de las desconfianzas, temores y sospechas tradicionales. 5) Asimetrías económicas que perturban las relaciones en tanto involucran a sectores - como el comercio - que ejercen presiones de muy diferentes maneras. 6) Caos en el mercado laboral regional que genera conflictos ante migraciones no reguladas y que se producen por asimetrías salariales. Esto lleva en algunos casos a manifestaciones de xenofobia y racismo, que si bien son solo incipientes, por ahora, representan un peligro a considerar con preocupación. 7) La identificación de agricultores intrusos como extranjeros (zona argentina del Río Uruguay). 8) La indiferencia de capas de la población campesina por causas muy específicas 
| 178 |

Processos transnacionales en las regiones de frontera:...

(el caso de las "brasiguayos" en el oriente de Paraguay). En este ejemplo debemos matizar diciendo que los comportamientos sociales de esta población inmigrante han variado notablemente frente a la crisis y que ahora sus contactos y relaciones con las organizaciones de agricultores paraguayos han cambiado a favor de su incorporación.

Nuestro seguimiento de las acciones de los movimientos nos llevo a la conclusión que los temas mas convocantes eran los relacionados a la tierra, la agricultura, la agroecológica, los campesinos sin tierra y sin títulos, etc. Así surgió “Los movimientos sociales en la integración regional: relaciones transfronterizas de solidaridad y lucha. La cuestión agraria integral. (Argentina- Brasil y Paraguay), 1999-2000", que surgió como un corolario de los factores detectados, y focalizó su interés en una cuestión central que aparecía - nítidamente - como dominante en las propuestas y acciones de los propios movimientos: la cuestión agraria integral y en particular las luchas por la tierra.

Al hablar de la cuestión agraria integral me estoy refiriendo a las luchas destinadas, no solo a obtener el acceso y control de la tierra, sino a otras cuestiones directamente vinculadas, como : la resistencia contra las represas hidroeléctricas, la defensa de los ecosistemas, la recuperación de técnicas tradicionales (sobre todo de agricultura orgánica) la incorporación de nuevas tecnologías apropiadas, la facilitación del acceso a los mercados, la instalación de ferias francas, y muchos otros temas vinculados a la vida y la producción rural en el ámbito de los pequeños productores y campesinos. Y, sobre todo a la defensa de una forma de vida con un rechazo explícito a las emigraciones. Los agricultores saben que ya no pueden migrar a las ciudades en busca de una vida mejor porque hay desempleo y el riesgo cierto de terminar en "villas miseria" o "favelas".

Debo aclarar que nuestro interés principal no fue realizar un estudio sobre la problemática agraria como tal sino efectuar una investigación sobre la manera en que la cuestión agraria regional se convirtió en un centro atractor de luchas sociales por sobre las divisiones nacionales fronterizas. En otras palabras, el foco de 
atención siguió siendo la integración regional y fronteriza en sus formas emergentes. Debo aquí hacer un salto para pasar al ultimo ciclo de investigaciones que comenzaron a realizarse bajo el título de "Procesos transfronterizos complejos: el caso de la Triple Frontera. Movimientos sociales, "grupos bajo sospecha, control y políticas públicas" y se extiende desde el 2003 al 2005".

Este proyecto se propuso conocer la enorme complejidad de la llamada Triple Frontera, que, por razones obvias, ha alcanzado un lugar de relieve en los medios de comunicación mundiales. Nos propusimos, en este caso, denunciar la simplificación grosera que se hace de esta realidad multiforme, la distorsión de la información que se "vende" como seria y fidedigna y la manipulación imperial de los poderes nacionales y locales con el fin de militarizar la zona con excusas pueriles y objetivos espurios.

Llegamos así a la nuestras investigaciones actuales en las que tratamos de dilucidar, después del 11 de setiembre, que lugar ocupa la Triple Frontera en el sistema mundo; en el sistema imperial y en esa búsqueda trabajamos en la organización del Foro de la Triple Frontera destinado a generar una conciencia local sobre las acechanzas que una zona tan estratégica y tan rica. La Triple Frontera posee la represa mas grande del mundo; reservas de biosfera de valor incalculable, el segundo acuífero mas grande del planeta, recursos turísticos de primer magnitud; un comercio de miles de millones en divisas anualmente, una población que creció de manera exponencial, universidades y centros tecnológicos de gran nivel; y sobre todo, algo que preocupa a quienes no ven con buenos ojos la integración de America Latina: tres sociedades que coexisten en paz a pesar de las estigmatizaciones permanentes. Es cierto que hay actividades ilícitas y posiblemente en un volumen preocupante - que deben ser investigadas antropológicamente pero no es menos cierto que eso no es la triple frontera, es solo una parte menor de una zona en expansión y desarrollo.

En síntesis, podemos afirmar que la región de fronteras, denominada triple frontera, es imposible de ser conocida mediante modelos simplificadores que desconozcan la gran complejidad de ese universo en el cual se producen fenómenos emergentes 
$\mid 180$ |

Processos transnacionales en las regiones de frontera:...

producto de la integración y de la respuesta local a las presiones internacionales ejercidas por el sistema imperial, las redes financieras globalizadas, las amenazas intervensionistas, y la voracidad del poder hegemónico mundial ante las riquezas inconmensurables de la zona, que además, posee un carácter geopolítico estratégico.

\section{Bibliografia}

ABÍNZANO, R. C. Regiones de frontera y procesos transfronterizos. In: Actas del Encuentro Internacional de Ciudades Fronterizas del Mercosur. Mercociudades, Municipalidad de Asunción. Asunción, octubre del 2003.

Mercosur: Un Modelo de Integración. Crítica y Compilación Documental. Editorial de la Universidad Nacional de Misiones. Serie Contemporánea. Tercera edición, actualizada con nuevo prefacio, 2001.

. Las instituciones en el proceso de integración. In: Primer Encuentro Rural Fronterizo. Central Única de Trabajadores de Brasil, (CUT), Porto Alegre, 1990.

Integración regional en áreas de frontera: Argentina, Brasil y Paraguay. Los movimientos sociales. In: Revista del Centro de Estudios Latinoamericanos. Universidad de Varsovia, Warzawa, 2001.

. Integración regional fronteriza "desde abajo". Nuevos sujetos colectivos, ¿nuevos poderes?. Estudios Regionales, año 9, num.17, nov. de 2001. Posadas. Sec. De Inv, y Postgrado. FHYCS, UNaM. Sevilla, 2000.

Los estudios antropológicos en los espacios transfronterizos. Publicación en CD con las Actas de la reunión de la Asociación de Estudios de Poblacion de Argentina (AEPA), 2002. 
Integración regional fronteriza: sectores vulnerables frente al impacto global. Políticas y acciones alternativas. Comunicación. En: ACTAS del III Encuentro de Científicos Sociales. op. cit., 1995.

AMIR, S. Mas allá del capitalismo senil. Por un siglo XXI no norteamericano. Buenos Aires, Paidos, 2005.

BAGGIO, L. La integración cultural entre ciudades fronterizas desde los municipios y las universidades. In: Actas del Encuentro Internacional de Ciudades Fronterizas del Mercosur. Mercociudades, Municipalidad de Asunción. Asunción, octubre del 2003.

BECKER, B. K. Geografia política e gestão do território no liminar do Século XXI. In. Revista brasileira de geografia. Vol. 53 N. 3, jul-set. IBGE Rio de Janeiro, 1991.

BELATO, D., ABÍNZANO, R.C., TREVISOL, J. V., y MOREIRA, S. C., Os impactos da Globalizaçao na nossa region. Mesa redonda de apertura. In: Anais do IV Encontro de Cientistas Socias, op. cit., 1996.

BOISER, S. Las regiones como espacios socialmente construidos. Revista de Naciones Unidas, CEPAL, N. 35, 1988.

BOX, LOUK y DE LA RIVE, B. ¿Sociedad fronteriza o frontera social?). In: Boletín de Estudios Latinoamericanos y del Caribe, Nro. 46, junio 1989.

CHOMSKY, N. La conquista continúa. 500 años de genocidio imperialista. Terramar, Caronte Ensayos, La Plata, 2007. . El miedo a la democracia. Barcelona, Grijalbo, 1992.

Hegemonía y supervivencia. La estrategia imperialista de los Estados Unidos. Barcelona, Liberduplex, 2005.

Piratas y emperadores. Terrorismo internacional en el mundo de hoy. 2004. 
CLAVAL, P. Espacio y poder. México, FCE, 1982.

ELIAS, N. Sociología fundamental. Barcelona, Gedisa, 1997.

FLECHA, A. O. Paraguay en la coyuntura económica actual. Asunción, E.T.I., s/f.

FOGEL, R. La ciencia y la tecnología en Paraguay. Su impacto socioambiental. Asunción, CERI, 1994.

FOGEL, R. Los campesinos sin tierra en la frontera. Asunción. Comité de Iglesias, 1990.

Desintegración social e integración en regiones fronterizas. In. Actas del Encuentro Internacional de Ciudades Fronterizas del Mercosur. Mercociudades, Muncipalidad de Asunción. Asunción, octubre del 2003.

FOUCAULT, M. Defender la sociedad. Curso en el College de France (1975-1976) Bs.As., FCE, 2001.

GALEANO, L (Comp.) Mujer y trabajo en el Paraguay. Asunción, Centro Paraguayo de Estudios Sociologicos, 1982.

GODELIER, M. Lo ideal y lo material. Pensamiento, economía, sociedades. Madrid, Taurus, 1989.

GOHN, M. da G. Os Sem-terra, ONGs e Cidadania. São Paulo, Cortez, 1997. . Teoria dos Movimentos Sociais. São Paulo, Loyola, 1997.

. História dos movimentos e lutas sociais. Construção da cidadania dos brasileiros. São Paulo, Loyola, 1995.

HEISFELD, A. O pensamento geopolítico no Brasil e na Argentina: um estudo analítico a partir do Barão do Rio Branco e de Estanislao Zeballos. En: IBIDEM.

HOBSBAWM, E. Naciones y nacionalismo. Barcelona, Crítica, 1995.

LATIN AMERICAN NEWSLETTERS. Servicio de Cartas informativas. Cincuenta anuales. Colección de los años 1989 a 1998. Londres, Inglaterra. 
IBARRA, P y TEJERINA, B. Comp. Los movimientos sociales. Transformaciones políticas y cambio cultural. Valladolid, Trotta, 1998.

ITURRIZA, J. y otros La integración y cooperación fronteriza en el programa Argentina-Brasil. Buenos Aires, BID-INTAL, 1991.

JIMENEZ NUÑES, A. El fenómeno de frontera y sus variables. Notas para una tipología. California, Universidad de Baja California, 1998.

JIMENEZ NUÑES, A. La frontera en América: observaciones, críticas y sugerencias. Sevilla, Escuela de Estudios Hispano-americanos y Univ. De Sevilla, 1997.

MACHADO DE OLIVEIRA, T. C. Comp. Territorios sin límites. Estudios sobre fronteras. Campo Grande, UFMS Editora, 2005.

Tipología de las relaciones relaciones transfronterizas. Elementos para un debate teórico práctico. In: Machado de Oliveira, Tito C. Comp. In: Territorios sin límites. Estudios sobre fronteras. Campo Grande, UFMS Editora, 2005.

MAEDER, E. Breve historia del nordeste argentino en su relación con el Paraguay y Rio Grande Do Sul. In: Revista de Estudios Regionales, A; o 1, num. 2, Corrientes, dic.1977.

MAM (Movimiento Agrario Misionero). Encuentro regional de pequeños y medianos productores del N.E.A., Oberá, agosto de 1990.

MARINI, R. M. El experimento neo-liberal en Brasil. In: Nueva Sociedad, nov. de 1993.

OLIVEIRA DE, A. A geografia das lutas no campo. Conflitos e violência, movimentos sociais e resistência a "nova república"'e a reforma agrária. Editora da Universidade de São Paulo, 1993.

OLIVEIRA, N. Áreas de fronteira na perspectiva da integração latinoamericana. In: Analise Coyuntural. N. 3, 3otrimestre, vol. 20, Porto Alegre, 1992. 
OVIEDO, A y, J. Misiones en el intercambio económico regional y en el contexto del Mercosur. Estudios Regionales. N.10. Sec. de Investigación y Postgrado, FHCS-UNaM, Poadas, 1997.

PASTORE, C. La lucha por la tierra en Paraguay. Montevideo, Antequera, 1972.

PETRAS, J. Clase, estado y poder en el tercer mundo. Casos de conflictos de clases en América Latina. Buenos Aires, FCE, 1993.

REYNOSO, C. Redes sociales y complejidad. Modelos interdisciplinarios en la gestión sostenible de la sociedad y la cultura. Bueno Aires, Colección Complejidad Humana, 2011.

ROFMAN, A. Estrategias alternativas frente al desafío del MERCOSUR. In: Pablo CICOLLELA y otros. Modelos de Integración en America Latina. Desafíos y alternativas en la construcción de un nuevo territorio latinoamericano. Buenos Aires, Centro Editor de América Latina, 1993.

ROJAS A, F. y otros. Integración regional y globalización. (Dossier) In: Nueva Sociedad, nº 125, Caracas, mayo-junio de 1993.

SADER, E y GENTILI, P (comp.) La trama del liberalismo. Mercado Crisis y exclusión social. Buenos Aires, EUDEBA, 2001.

SANTOS, B. de S. Para descolonizar occidente. Mas Allá Del pensamiento abismal. Buenos Aires, CLACSO, UBA, Prometeo, FFYL, 2010.

SANTOS, M. De la totalidad al lugar. Barcelona, Oikos-Tau, 1996.

SASSEN, S. Sociología de la globalización. Buenos Aires, Katz, 2007.

SCHIAVONI, L. Pesadas cargas, fragiles pasos. Posadas, Edit. Universitaria, 1993.

SCHIAVONI, L. Pobreza y fronteras. In: Actas del Encuentro Internacional de Ciudades Fronterizas del Mercosur. Mercociudades, Municipalidad de Asunción. Asunción, octubre del 2003. Tercer Encuentro Internacional de Organizaciones Rurales ArgentinaBrasil y Paraguay. Campinas das Missões, Rio Grande Do Sul, 15 al 17 de febrero de 1991 (actas). 
THOMPSON, E. P. Historia Social y Antropología. Mexico, Instituto Mora, 1994.

VITALE, L. Introducción a una teoría de la história para America Latina. Buenos Aires, Planeta, 1992.

WALLERSTEIN, I. Despues del liberalismo, Mexico, Siglo XXI, 1996.

. Análisis de los sistemas mundiales. En: Giddens, A. La teoría social hoy. Alianza, Madrid, 1990.

La de decadencia del imperio. EEUU en un mundo caótico. Tafalla, Txalaparta, 2005. 